\title{
[gw22-e0603] SHORT-TERM AND LONG-TERM OUTCOME OF EARLY INVASIVE THERAPY IN PATIENTS WITH NON-ST-SEGMENT ELEVATION ACUTE CORONARY SYNDROME: A META-ANALYSIS
}

Yu Zixiang, Ma Yitong, Yang Yining, Liu Fen Department Of Cardiology, First Affiliated Hospital, Xinjiang Medical University, Urumqi, China

10.1136/heartjnl-2011-300867.325

Objective To determine the short-term and long-term outcome of an invasive compared to a conservative strategy for treating UA/NSTEMI.

Methods We searched The Cochrane Library (Issue 1.2011), MEDLINE (1996 to September 2010), EMBASE (1974 to September 2010), CBM (1989 to 2011), CNKI (1997 to 2011), VIP (1989 to 2011). The quality of included studies was critically evaluated. Data analyses were performed with The Cochrane Collaboration's Revman 5.0 software.

Results Seven randomised controlled trials met the including criteria. The meta-analysis showed the incidence of all-cause mortality at 6 months follow-up was lower in the early invasive group compared with the conservative group (RR $0.75,95 \% \mathrm{CI}$ 0.61 to $0.92, p=0.007$ ), there was a non-significant trend toward more deaths (RR 0.88, 95\% CI 0.65 to $1.18, p=0.38$ ); Myocardial infarction were significantly decreased by an invasive strategy with RR of 0.74 (95\% CI 0.63 to 0.87 ); there was a reduction in rehospitalisation for unstable angina (RR 0.66, 95\% CI 0.61 to $0.73, p<0.00001)$; The invasive strategy was associated with a twofold increase in the RR of PCI-related myocardial infarction (as variably defined). There was a non-significant increase in bleeding by an invasive strategy, but, a routine invasive strategy was associated with a significantly higher bleeding rate at 1-year follow-up (RR 2.22, 95\% CI 1.55 to 3.17, p<0.0001). Over 5 years, The most marked treatment effect was on MI $(10.0 \%$ RI strategy vs $12.9 \%$ SI strategy), and there were consistent trends for cardiovascular deaths (RR 0.83, 95\% CI 0.68 to $1.01, \mathrm{p}=0.068$ ) and All-cause deaths (RR 0.90, 95\% CI 0.77 to 1.05 ). There was an $11.1 \%$ absolute risk reduction in highest-risk patients.

Conclusion An early invasive strategy is preferable to a conservative strategy in the treatment of UA/NSTEMI at longterm follow-up, especially in the high risk patients. 